QUARTERLY OF APPLIED MATHEMATICS

VOLUME LXVII, NUMBER 3

SEPTEMBER 2009, PAGES 489-502

S 0033-569X(09)01156-7

Article electronically published on May 6, 2009

\title{
FROM A MULTIDIMENSIONAL QUANTUM HYDRODYNAMIC MODEL TO THE CLASSICAL DRIFT-DIFFUSION EQUATION
}

\author{
BY
}

YEPING LI

Department of Mathematics, Shanghai Normal University, Shanghai 200234, China

\begin{abstract}
In the paper, we discuss the combined semiclassical and relaxation-time limits of a multidimensional isentropic quantum hydrodynamical model for semiconductors with small momentum relaxation time and Planck constant. The quantum hydrodynamic equations consist of the isentropic Euler equations for the particle density and current density including the quantum Bohn potential and a momentum relaxation term. The momentum equation is highly nonlinear and contains a dispersive term with thirdorder derivatives. The equations are self-consistently coupled to the Poisson equation for the electrostatic potential. With the help of the Maxwell-type iteration, we prove that, as the Planck constant and the relaxation time tend to zero, periodic initial-value problems of a scaled isentropic quantum hydrodynamic model have unique smooth solutions existing in the time interval where the classical drift-diffusion models have smooth solutions.
\end{abstract}

1. Introduction. In the present paper, we consider the initial value problem of the quantum hydrodynamic model for semiconductors where an additional relaxation term is involved in the linear momentum equation to model the interaction between the electron and crystal lattice. The rescaled multidimensional quantum hydrodynamic models for semiconductors (see [5, 6]) are then given by

$$
\left\{\begin{array}{l}
\partial_{t} n+\frac{1}{\epsilon} \nabla \cdot(n u)=0, \\
\partial_{t}(n u)+\frac{1}{\epsilon} \nabla \cdot(n u \otimes u+p(n))=\frac{n \nabla \phi}{\epsilon}+\frac{h^{2}}{4 \epsilon} \nabla \cdot\left(n \nabla^{2}(\log n)\right)-\frac{n u}{\epsilon^{2}}, \\
\lambda^{2} \Delta \phi=n-b(x) .
\end{array}\right.
$$

The variables are the electron density $n$, the mean velocity $u$, and the electrostatic potential $\phi . \quad p(n)$ is the given strictly increasing function and denotes the pressure. The function $b(x)$ stands for the prescribed density of positive charged background ions (doping profile). The parameters are the (scaled) Planck constant $h$, the momentum relaxation time $\epsilon$, and the Debye length $\lambda$. The quantum hydrodynamic equations can

Received February 17, 2008.

2000 Mathematics Subject Classification. Primary 35B25, 35M20, 35L45.

Key words and phrases. Relaxation limit, isentropic quantum models semiconductors, energy estimates. E-mail address: ypleemei@yahoo.com.cn 
be interpreted as Euler equations for a charged isentropic gas, containing the nonlinear dispersive term $\frac{h^{2}}{4} \nabla \cdot\left(n \nabla^{2}(\log n)\right)$ and the relaxation term $\frac{n u}{\epsilon^{2}}$. Moreover,

$$
\frac{h^{2}}{4} \nabla \cdot\left(n \nabla^{2}(\log n)\right)=\frac{h^{2}}{2} n \nabla\left(\frac{\Delta \sqrt{n}}{\sqrt{n}}\right),
$$

and $\frac{\Delta \sqrt{n}}{\sqrt{n}}$ is the quantum Bohm potential. Note that the scaling

$$
t=\epsilon \tilde{t}
$$

converts (1.1) back into the original quantum isentropic model in [7, 8] with $\tilde{t}$ as its time variable. The scaled-time variable $t$ was first introduced in 22 to study the relation between the classical hydrodynamical models and corresponding drift-diffusion models.

Since we are interested in the combined semiclassical and relaxation limits, we can take $\lambda=1, h=\epsilon$. On the other hand, the operator $\nabla \Delta^{-1}$ is a bound linear operator on $L^{2}\left(\mathbb{T}^{3}\right)$, where and in the sequel, $\mathbb{T}^{3}$ is the three-dimensional unit torus $(0,1] \times(0,1] \times$ $(0,1]$. It is convenient to make use of the variable transformation $n=w^{2}$ as in [11, 12] and the enthalpy $f(n)$ satisfying $f^{\prime}(n)=\frac{p^{\prime}(n)}{n}$. With the above simplifications, we can rewrite the model (1.1) for smooth solutions as

$$
\left\{\begin{array}{l}
w_{t}+\frac{1}{2 \epsilon} w \operatorname{div} u+\frac{1}{\epsilon} \nabla w \cdot u=0, \\
u_{t}+\frac{1}{\epsilon}(u \cdot \nabla) u+\frac{1}{\epsilon} \nabla f\left(w^{2}\right)=\frac{1}{\epsilon} \nabla \Delta^{-1}\left(w^{2}-b(x)\right)+\frac{\epsilon}{2} \nabla\left(\frac{\Delta w}{w}\right)-\frac{u}{\epsilon^{2}} .
\end{array}\right.
$$

Recently, many efforts have been made on the quantum hydrodynamic (Euler-Poisson) system. More precisely, the existence and uniqueness of thermal equilibrium steadystate classical solutions for one and high dimensional quantum models have been studied in [3, 24]. The corresponding nonthermal equilibrium steady-state solutions have also been considered in [4, 9, 27] for general monotonic pressure functions and in 14] for general pressure functions, however, with different boundary conditions, for instance, assuming Dirichlet data for the velocity potential [9] or employing nonlinear boundary conditions [27]. For the time-dependent system, the local- and global-in-time existence of the classical solutions was obtained in a bounded domain [12, 15] (subject to boundary conditions on the density and the electrostatic potential) and on the real line [11, for ir-rotational flow in the whole space 20] assuming strictly convex pressure functions and in the multidimensional torus $\mathbb{T}^{n}$ [19], while paper [2] established the global existence of finite energy weak solutions to the quantum hydrodynamic system for arbitrarily large data.

Relaxation limits in the classical hydrodynamic equations have been performed extensively. In one space dimension, the relaxation limit problem for isentropic hydrodynamic models has been investigated in the compactness frameworks for nonsmooth solutions of conservation laws; see [22]. Paper [10] also discussed similar results for the one-dimensional nonisentropic hydrodynamic model for semiconductors with zero thermal conductivity. In [18, 17], the authors considered the multidimensional isentropic unipolar hydrodynamic model and the corresponding bipolar model with $x$ in a bounded domain, assumed the existence of $L^{\infty}$-solutions in a $\tau$-independent time interval, and justified the relaxation limit in a compactness framework for nonsmooth solutions. In [26, 21, the authors studied the diffusive relaxation of multidimensional isentropic and 
nonisentropic hydrodynamical models for semiconductors by the Maxewell iteration. Finally, paper [12] first investigated the relaxation limit for the quantum hydrodynamic models, where the relaxation-time limits both in the stationary and in the transient case are proved. Similar results about classical hydrodynamic models for semiconductors have been extensively discussed in [1, 16, 28. However, the combined semiclassical and relaxation limit for the quantum hydrodynamical model for semiconductors is not studied. Motivated by [12, 26, 21, we expect that the quantum hydrodynamic model and the classical drift-diffusion model give similar results when $\epsilon$ is small, which can be seen formally as follows. Applying the Maxwell-type iteration to the momentum equations in (1.2) gives

$$
\begin{aligned}
u & =-\epsilon \nabla f\left(w^{2}\right)+\epsilon \nabla \Delta^{-1}\left(w^{2}-b(x)\right)-\epsilon(u \cdot) u+\frac{\epsilon^{3}}{2} \nabla\left(\frac{\Delta w}{w}\right)-\epsilon^{2} \partial_{t} u \\
& =-\epsilon \nabla f\left(w^{2}\right)+\epsilon \nabla \Delta^{-1}\left(w^{2}-b(x)\right)+O\left(\epsilon^{2}\right) .
\end{aligned}
$$

Substituting these truncations $u=-\epsilon \nabla f\left(w^{2}\right)+\epsilon \nabla \Delta^{-1}\left(w^{2}-b(x)\right)$ into the mass equations in (1.2), we arrive at the classical drift-diffusion model

$$
2 w \partial_{t} w-\operatorname{div}\left(\nabla p\left(w^{2}\right)-w^{2} \nabla \Delta^{-1}\left(w^{2}-b(x)\right)\right)=0 ;
$$

this is a parabolic-type system (see [13, 23]), provided that $p^{\prime}\left(w^{2}\right)>0$. Now we state our main results in the following.

Theorem 1.1. Suppose $f\left(w^{2}\right) \in C^{8}(0, \infty), f^{\prime}\left(w^{2}\right)>0, b(x) \in H^{6}\left(\mathbb{T}^{3}\right)$, and that the quantum drift-diffusion model (1.3) with the initial data $w(x, 0)=w_{0}(x)>0$ has a solution $w \in C\left(\left[0, T_{*}\right], H^{8}\left(\mathbb{T}^{3}\right)\right) \cap C^{1}\left(\left[0, T_{*}\right], H^{7}\left(\mathbb{T}^{3}\right)\right)$ with a positive lower bound.

Then, for $\epsilon$ sufficiently small, the quantum isentropic model (1.2) with periodic initial data

$$
\left\{\begin{array}{l}
w(x, 0)=w_{0}(x) \\
u(x, 0)=\epsilon \nabla \Delta^{-1}\left(w^{2}(x, 0)-b(x)\right)-\epsilon \nabla f\left(w_{0}^{2}\right)
\end{array}\right.
$$

has a unique solution $\left(w^{\epsilon}, u^{\epsilon}\right) \in C\left(\left[0, T_{*}\right], H^{6}\left(\mathbb{T}^{3}\right)\right) \times C\left(\left[0, T_{*}\right], H^{5}\left(\mathbb{T}^{3}\right)\right)$, and there exists a constant $K>0$, independent of $\epsilon$ but dependent on $T_{*}(<\infty)$, such that

$$
\sup _{t \in\left[0, T_{*}\right]}\left(\left\|w^{\epsilon}-w_{\epsilon}\right\|_{4}+\epsilon\left\|\partial_{x}^{5}\left(w^{\epsilon}-w_{\epsilon}\right)\right\|_{1}+\left\|u^{\epsilon}-u_{\epsilon}\right\|_{5}\right) \leq K \epsilon^{2}
$$

where

$$
w_{\epsilon}=w, \quad u_{\epsilon}=\epsilon \nabla \Delta^{-1}\left(w^{2}-b(x)\right)-\epsilon \nabla f\left(w^{2}\right) .
$$

To prove this result, we shall adopt and modify the arguments in [25, 26]. More precisely, we first construct the approximate solutions, based on the existence of smooth solutions for the corresponding classical drift-diffusion model in [13, 23. Next, we establish the continuation principle (convergence-stability lemma) of the Cauchy problem, under the assumption of the important a priori estimate (which is indeed referred to as a convergence assumption in [25, 26]). Finally, we should show the a priori convergence assumption; namely, from the energy methods and the special nonlinear structure of the quantum hydrodynamic models, we can obtain the a priori estimate. However, we have to face up to two difficulties, contrasted with [25, 26]. The first one is from the 
Bohm potential, which is a third-order dispersive term. Our strategy is to reformulate the conservation law of mass in the quantum hydrodynamic equation as a fourth-order wave equation as in [11, 12. The second difficulty is that we cannot directly make use of the previous convergence-stability lemma, which was first formulated by W.A. Yong in [25], since it only fits into the symmetrizable hyperbolic system with relaxation term. So here we have to establish the corresponding convergence-stability lemma for our quantum hydrodynamic model with the third-order dispersive term.

REMARK 1.2. Our conclusion implies that if the classical drift-diffusion model has a global smooth solution on $[0, \infty]$ with $w$ having a positive lower bound, then there exists $\epsilon_{0}>0$ such that the quantum isentropic hydrodynamical model has a unique smooth solution up to the time $\infty$ when $\epsilon<\epsilon_{0}$, and when $T<\infty$,

$$
\left(w^{\epsilon}, u^{\epsilon}\right)=\left(w_{\epsilon}, u_{\epsilon}\right)+O\left(\epsilon^{2}\right) .
$$

Moreover, we believe that our result also holds for the more general multidimensional $(d>3)$ quantum isentropic hydrodynamic model for semiconductors.

REMARK 1.3. As far as we know, this is the first time that the convergence-stability lemma of the symmetrizable hyperbolic system has been extended to that of the nonhyperbolic case, and we apply it to investigate the combined semiclassical and relaxation limits for the multi-dimensional quantum isentropic hydrodynamic model for semiconductors.

REMARK 1.4. Employing similar arguments, we can investigate the combined semiclassical and relaxation limits for the bipolar quantum hydrodynamic model for semiconductors. Moreover, on the basis of the convergence-stability lemma in this paper, we can use the matched expansion method to discuss these limits for the relaxation limit for the quantum hydrodynamic semiconductor model with more general initial data.

This paper is organized as follows. In section 2 we are going to derive the convergencestability result and construct the formal approximation (1.6). In section 3 we prove the validity of the formal approximation and conclude the existence of the solution to $\left(w^{\epsilon}, u^{\epsilon}\right)$ in the time interval where $w$ is well-defined.

2. The convergence-stability lemmas and formal approximations. In this section, we are going to derive convergence-stability lemmas and construct formal approximate solutions. To begin with, for

$$
\left\{\begin{array}{l}
w_{t}+\frac{1}{2 \epsilon} w \operatorname{div} u+\frac{1}{\epsilon} \nabla w \cdot u=0, \\
u_{t}+\frac{1}{\epsilon}(u \cdot \nabla) u+\frac{1}{\epsilon} \nabla f\left(w^{2}\right)=\frac{1}{\epsilon} \nabla \Delta^{-1}\left(w^{2}-b(x)\right)+\frac{\epsilon}{2} \nabla\left(\frac{\Delta w}{w}\right)-\frac{u}{\epsilon^{2}},
\end{array}\right.
$$

we have

Lemma 2.1 (see [1]). Assume that $f\left(w^{2}\right) \in C^{8}(0,+\infty), f^{\prime}\left(w^{2}\right)>0, b(x) \in H^{6}\left(\mathbb{T}^{3}\right)$ and that $(w(x, 0), u(x, 0)) \in H^{6}\left(\mathbb{T}^{3}\right) \times H^{5}\left(\mathbb{T}^{3}\right), w(x, 0)>0$. Then the unique classical solution $(w, u)$ of the system (2.1) with the initial data $(w(x, 0), u(x, 0))$ exists for $t \in[0, T]$ and satisfies $w>0$ in $\mathbb{T}^{3} \times[0, T]$ and

$$
w \in C^{i}\left([0, T] ; H^{6-2 i}\left(\mathbb{T}^{3}\right)\right), \quad i=0,1,2, \quad u \in C^{j}\left([0, T] ; H^{5-2 j}\left(\mathbb{T}^{3}\right)\right), \quad j=0,1 .
$$


Fixing $\epsilon$, and according to Lemma 2.1, there is a time interval $[0, T]$ so that (2.1) has a unique $H^{6} \times H^{5}$ solution

$$
\left(w^{\epsilon}, u^{\epsilon}\right) \in C\left([0, T], H^{6}\left(\mathbb{T}^{3}\right)\right) \times C\left([0, T], H^{5}\left(\mathbb{T}^{3}\right)\right) .
$$

Define

$$
T_{\epsilon}=\sup \left\{T>0:\left(w^{\epsilon}, u^{\epsilon}\right) \in C\left([0, T], H^{6}\left(\mathbb{T}^{3}\right)\right) \times C\left([0, T], H^{5}\left(\mathbb{T}^{3}\right)\right)\right\} .
$$

Namely, $\left[0, T_{\epsilon}\right)$ is the maximal time interval of $H^{6}\left(\mathbb{T}^{3}\right) \times H^{5}\left(\mathbb{T}^{3}\right)$-existence. Note that $T_{\epsilon}$ may tend to zero as $\epsilon$ goes to a certain singular point, say 0 .

In order to show that $\lim _{\epsilon \rightarrow 0} T_{\epsilon}>0$, we need the convergence-stability lemma (see [25, 26]). That is,

Lemma 2.2. Let $b(x) \in H^{6}\left(\mathbb{T}^{3}\right)$ and $b(x)>0$, and let $\left[0, T_{\epsilon}\right)$ be the maximal time interval such that (1.2) with $(w, u)(x, 0)=(\bar{w}, \bar{u})(x, \epsilon) \in H^{6}\left(\mathbb{T}^{3}\right) \times H^{5}\left(\mathbb{T}^{3}\right)$ for all $(x, \epsilon)$ and $\bar{w}(x, \epsilon)>0$ has a unique solution $\left(w^{\epsilon}, u^{\epsilon}\right) \in C\left(\left[0, T_{\epsilon}\right], H^{6}\left(\mathbb{T}^{3}\right)\right) \times C\left(\left[0, T_{\epsilon}\right], H^{5}\left(\mathbb{T}^{3}\right)\right)$ satisfying $w^{\epsilon}(x, t)>0$. Assume there exists $T_{*}>0$ and $\left(w_{\epsilon}, u_{\epsilon}\right) \in H^{6}\left(\mathbb{T}^{3}\right) \times H^{5}\left(\mathbb{T}^{3}\right)$ for each $\epsilon$, satisfying

$$
w_{\epsilon}(x, t)>0
$$

such that it follows that for $t \in\left[0, \min \left\{T_{*}, T_{\epsilon}\right\}\right)$, as $\epsilon$ tends to the singular point,

$$
\begin{aligned}
& \sup _{x, t}\left|\left(w^{\epsilon}, u^{\epsilon}\right)(x, t)-\left(w_{\epsilon}, u_{\epsilon}\right)(x, t)\right|=o(1), \\
& \sup _{t}\left(\left\|w^{\epsilon}(x, t)-w_{\epsilon}(x, t)\right\|_{6}+\left\|u^{\epsilon}(x, t)-u_{\epsilon}(x, t)\right\|_{5}\right)=O(1) .
\end{aligned}
$$

Then

$$
T_{\epsilon}>T_{*}
$$

for all $\epsilon$ in a neighborhood of the singular point.

Proof. Otherwise, there is a sequence $\left\{\epsilon_{k}\right\}_{k \geq 1}$ such that $\lim _{k \rightarrow \infty} \epsilon_{k}=0$ and $T_{\epsilon_{k}} \leq T_{*}$. Thanks to the convergence assumption, there exists $w^{\epsilon_{k}}>0$. On the other hand, we deduce from

$$
\left\|w^{\epsilon_{k}}\right\|_{6}+\left\|u^{\epsilon_{k}}\right\|_{5} \leq\left\|w^{\epsilon_{k}}-w_{\epsilon_{k}}\right\|_{6}+\left\|u^{\epsilon_{k}}-u_{\epsilon_{k}}\right\|_{5}+\left\|w_{\epsilon_{k}}\right\|_{6}+\left\|u_{\epsilon_{k}}\right\|_{5}
$$

and the convergence assumption that $\left\|w^{\epsilon_{k}}\right\|_{6}+\left\|u^{\epsilon_{k}}\right\|_{5}$ is bounded uniformly with respect to $t \in\left[0, T_{\epsilon_{k}}\right.$ ). Now we could apply Lemma 2.1 , beginning at a time $t$ less than $T_{\epsilon_{k}}$, to continue this solution beyond $T_{\epsilon_{k}}$. This contradicts the definition of $T_{\epsilon}$. This completes the proof.

In the following, we prepare to construct the approximation $\left(w_{\epsilon}, u_{\epsilon}\right)$ as above. Let $w$ solve the IVP of the classical drift-diffusion model (see [13, 23]), i.e.,

$$
\left\{\begin{array}{l}
2 w \partial_{t} w-\operatorname{div}\left(\nabla p\left(w^{2}\right)-w^{2} \nabla \Delta^{-1}\left(w^{2}-b(x)\right)\right)=0 \\
w(x, 0)=w_{0}(x)
\end{array}\right.
$$

Inspired by the Maxwell-type iteration, we take

$$
\left\{\begin{array}{l}
w_{\epsilon}=w \\
u_{\epsilon}=\epsilon \nabla \Delta^{-1}\left(w^{2}-b(x)\right)-\epsilon \nabla f\left(w^{2}\right) .
\end{array}\right.
$$


Defining

$$
\begin{aligned}
R= & \frac{\partial_{t} u_{\epsilon}+\left(u_{\epsilon} \cdot \nabla\right) u_{\epsilon} / \epsilon-\frac{\epsilon}{2} \nabla\left(\frac{\Delta w}{w}\right)}{\epsilon}=\partial_{t}\left(\nabla \Delta^{-1}\left(w^{2}-b(x)\right)-\nabla f\left(w^{2}\right)\right) \\
& +\left(\left(\nabla \Delta^{-1}\left(w^{2}-b(x)\right)-\nabla f\left(w^{2}\right)\right) \cdot \nabla\right)\left(\nabla \Delta^{-1}\left(w^{2}-b(x)\right)-\nabla f\left(w^{2}\right)\right)-\frac{1}{2} \nabla\left(\frac{\Delta w}{w}\right),
\end{aligned}
$$

we have

$$
\left\{\begin{array}{l}
w_{\epsilon t}+\frac{1}{2 \epsilon} w_{\epsilon} \operatorname{div} u_{\epsilon}+\frac{1}{\epsilon} \nabla w_{\epsilon} \cdot u_{\epsilon}=0 \\
u_{\epsilon t}+\frac{1}{\epsilon}\left(u_{\epsilon} \cdot \nabla\right) u_{\epsilon}+\frac{1}{\epsilon} \nabla f\left(w_{\epsilon}^{2}\right)=\frac{1}{\epsilon} \nabla \Delta^{-1}\left(w_{\epsilon}^{2}-b(x)\right)+\frac{\epsilon}{2} \nabla\left(\frac{\Delta w_{\epsilon}}{w_{\epsilon}}\right)-\frac{u_{\epsilon}}{\epsilon^{2}}+\epsilon R
\end{array}\right.
$$

From well-known calculus inequalities in Sobolev spaces and the regularity of $w$, we have the following regularity result on $\left(w_{\epsilon}, u_{\epsilon}\right)$ :

Lemma 2.3. Assume that $f\left(w^{2}\right) \in C^{8}(0, \infty), f^{\prime}\left(w^{2}\right)>0$, and $w \in C\left(\left[0, T_{*}\right], H^{7}\left(\mathbb{T}^{3}\right)\right) \cap$ $C^{1}\left(\left[0, T_{*}\right], H^{6}\left(\mathbb{T}^{3}\right)\right)$ has positive lower bound. Moreover, if $b(x) \in H^{6}\left(\mathbb{T}^{3}\right)$ and $b(x)>0$, then $u_{\epsilon} \in C\left(\left[0, T_{*}\right], H^{6}\left(\mathbb{T}^{3}\right)\right) \cap C^{1}\left(\left[0, T_{*}\right], H^{5}\left(\mathbb{T}^{3}\right)\right)$ and $R \in C\left(\left[0, T_{*}\right], H^{4}\left(\mathbb{T}^{3}\right)\right)$.

3. The proof of the a priori estimate. Thanks to Lemma 2.2, it suffices to prove the error estimates in (1.5) for $t \in\left[0, \min \left\{T_{*}, T_{\epsilon}\right\}\right)$ in this section. To begin with, in order to avoid the dispersive third-order term, we reformulate (1.2) 1 of the quantum hydrodynamic equation as a fourth-order wave equation. For this, differentiating (1.2) 1 with respect to time $t$ and replacing by $(1.2)_{2}$, we have

$$
\begin{array}{r}
w_{t t}+\frac{1}{w} w_{t}^{2}+\frac{1}{\epsilon^{2}} w_{t}-\frac{1}{2 \epsilon^{2} w} \Delta p\left(w^{2}\right)-\frac{1}{2 \epsilon^{2} w} \nabla^{2} \cdot\left(w^{2} u \otimes u\right) \\
+\frac{1}{2 \epsilon^{2} w} \operatorname{div}\left(w^{2} \nabla \Delta^{-1}\left(w^{2}-b(x)\right)\right)+\frac{1}{4} \Delta^{2} w-\frac{1}{4 w}(\Delta w)^{2}=0 .
\end{array}
$$

Similarly, we can derive the wave equation satisfied by $w_{\epsilon}$ as the following:

$$
\begin{gathered}
w_{\epsilon t t}+\frac{1}{w_{\epsilon}} w_{\epsilon t}^{2}+\frac{1}{\epsilon^{2}} w_{\epsilon t}-\frac{1}{2 \epsilon^{2} w_{\epsilon}} \Delta p\left(w_{\epsilon}^{2}\right)-\frac{1}{2 \epsilon^{2} w_{\epsilon}} \nabla^{2} \cdot\left(w_{\epsilon}^{2} u_{\epsilon} \otimes u_{\epsilon}\right) \\
+\frac{1}{2 \epsilon^{2} w_{\epsilon}} \operatorname{div}\left(w_{\epsilon}^{2} \nabla \Delta^{-1}\left(w_{\epsilon}^{2}-b(x)\right)\right)+\frac{1}{4} \Delta^{2} w_{\epsilon}-\frac{1}{4 w_{\epsilon}}\left(\Delta w_{\epsilon}\right)^{2}=-\frac{1}{2} \operatorname{div} R .
\end{gathered}
$$

Further, setting

$$
(\psi, \eta)^{*}=\left(w_{\epsilon}-w^{\epsilon}, u_{\epsilon}-u^{\epsilon}\right)^{*}
$$


from the equations in (1.2), (2.4), (3.1) and (3.2), it follows that the error $(\psi, \eta)$ satisfies

$$
\begin{aligned}
& \psi_{t t}+\frac{1}{\epsilon^{2}} \psi_{t}+\frac{1}{4} \Delta^{2} \psi+\frac{1}{w_{\epsilon}} w_{\epsilon t}^{2}-\frac{1}{w^{\epsilon}} w_{t}^{\epsilon 2}-\frac{1}{2 \epsilon^{2} w_{\epsilon}} \Delta p\left(w_{\epsilon}^{2}\right)+\frac{1}{2 \epsilon^{2} w^{\epsilon}} \Delta p\left(w^{\epsilon 2}\right) \\
+ & \frac{1}{2 \epsilon^{2} w_{\epsilon}} \operatorname{div}\left(w_{\epsilon}^{2} \nabla \Delta^{-1}\left(w_{\epsilon}^{2}-b(x)\right)\right)-\frac{1}{2 \epsilon^{2} w^{\epsilon}} \operatorname{div}\left(w^{\epsilon 2} \nabla \Delta^{-1}\left(w^{\epsilon 2}-b(x)\right)\right) \\
- & \frac{1}{2 \epsilon^{2} w_{\epsilon}} \nabla^{2} \cdot\left(w_{\epsilon}^{2} u_{\epsilon} \otimes u_{\epsilon}\right)+\frac{1}{2 \epsilon^{2} w^{\epsilon}} \nabla^{2} \cdot\left(w^{\epsilon 2} u^{\epsilon} \otimes u^{\epsilon}\right)-\frac{1}{4 w_{\epsilon}}\left(\Delta w_{\epsilon}\right)^{2} \\
+ & \frac{1}{4 w^{\epsilon}}\left(\Delta w^{\epsilon}\right)^{2}=-\frac{1}{2} \operatorname{div} R, \\
& \eta_{t}+\frac{1}{\epsilon^{2}} \eta+\frac{1}{\epsilon} \nabla f\left(w_{\epsilon}^{2}\right)-\frac{1}{\epsilon} \nabla f\left(w^{\epsilon 2}\right)+\frac{1}{\epsilon}\left(\left(u_{\epsilon} \cdot \nabla\right) u_{\epsilon}-\left(u^{\epsilon} \cdot \nabla\right) u^{\epsilon}\right) \\
= & \frac{1}{\epsilon} \nabla \Delta^{-1}\left(w^{\epsilon 2}-b(x)\right)-\frac{\eta}{\epsilon^{2}}-\frac{\epsilon}{2} \nabla\left(\frac{\Delta w_{\epsilon}}{w_{\epsilon}}\right)+\frac{\epsilon}{2} \nabla\left(\frac{\Delta w^{\epsilon}}{w^{\epsilon}}\right)+\epsilon R .
\end{aligned}
$$

Moreover, we also need

$$
\psi_{t}+\frac{1}{2 \epsilon} w^{\epsilon} \operatorname{div} \eta+\frac{1}{2 \epsilon} \psi \operatorname{div} u_{\epsilon}+\frac{1}{\epsilon} u^{\epsilon} \nabla \psi+\frac{1}{\epsilon} \eta \nabla w_{\epsilon}=0 .
$$

For the sake of clarity, we divide the following arguments into lemmas.

Lemma 3.1. Set

$$
D=D(t)=\frac{\|\psi\|_{H^{4}}+\epsilon\left\|\partial_{x}^{5} \psi\right\|_{H^{1}}+\|\eta\|_{H^{5}}+\epsilon\left\|\psi_{t}\right\|_{H^{4}}}{\epsilon} .
$$

Then we have

$$
\begin{aligned}
& \left|u^{\epsilon}\right|,\left|u_{x}^{\epsilon}\right|,\left|u_{x x}^{\epsilon}\right|,\left|u_{x x x}^{\epsilon}\right| \leq C \epsilon+C \epsilon D, \\
& \left|w_{t}^{\epsilon}\right|,\left|w_{t x}^{\epsilon}\right|,\left|w_{t x x}^{\epsilon}\right|,\left|w_{x x x}^{\epsilon}\right|,\left|w_{x x x x}^{\epsilon}\right| \leq C+C D, \\
& \left|w_{x}^{\epsilon}\right|,\left|w_{x x}^{\epsilon}\right| \leq C+C \epsilon D .
\end{aligned}
$$

Proof. It is obvious from Sobolev's imbedding theorem and Lemma 2.3 that

$$
\left|u^{\epsilon}\right| \leq\left|u^{\epsilon}-u_{\epsilon}\right|+\left|u_{\epsilon}\right| \leq C\left\|u^{\epsilon}-u_{\epsilon}\right\|_{5}+\left\|u_{\epsilon}\right\|_{5} \leq C \epsilon+C \epsilon D .
$$

Similarly, we can prove other estimates.

Lemma 3.2. Under the assumptions of Theorem 1.1, it follows that for $0 \leq \alpha \leq 4$,

$$
\begin{aligned}
& \frac{1}{2} \frac{d}{d t} \int\left(\partial_{x}^{\alpha} \eta\right)^{2} d x+\frac{15}{16 \epsilon^{2}} \int\left(\partial_{x}^{\alpha} \eta\right)^{2} d x \leq \frac{\left\|\partial_{x}^{\alpha} \psi_{t}\right\|^{2}}{2} \\
&+\quad C \epsilon^{4}+C\left(1+D^{10}\right)\left(\|\psi\|_{\alpha+1}^{2}+\|\eta\|_{\alpha}^{2}+\epsilon^{2}\left\|\partial_{x}^{\alpha} \Delta \psi\right\|^{2}\right) .
\end{aligned}
$$

Proof. Multiplying (3.4) by $\eta$ and integrating the resultant equation over $\Omega$, we have

$$
\begin{array}{r}
\frac{1}{2} \frac{d}{d t} \int \eta^{2} d x+\frac{1}{\epsilon^{2}} \int \eta^{2} d x+\frac{1}{\epsilon} \int(\nabla \\
=\int\left(\frac{1}{\epsilon} \nabla \Delta^{-1} \psi+\frac{1}{2 \epsilon} \nabla\left(\frac{\Delta w_{\epsilon}}{w_{\epsilon}}-\frac{\Delta w^{\epsilon}}{w^{\epsilon}}\right)+\epsilon R\right) \eta d x .
\end{array}
$$


From the Cauchy-Schwarz and Young inequalities, one has

$$
\begin{aligned}
& \frac{1}{2 \epsilon} \int\left(\left(u_{\epsilon} \cdot \nabla\right) u_{\epsilon}-\left(u^{\epsilon} \cdot \nabla\right) u^{\epsilon}\right) \eta d x+\frac{1}{\epsilon} \int \nabla \Delta^{-1}\left(w_{\epsilon}+w^{\epsilon}\right) \psi \eta d x+\int \epsilon R \eta d x \\
= & -\frac{1}{2 \epsilon} \int\left(\left(u_{\epsilon} \cdot \nabla\right) \eta-(\eta \cdot \nabla) u^{\epsilon}\right) \eta d x+\frac{1}{\epsilon} \int \nabla \Delta^{-1}\left(w_{\epsilon}+w^{\epsilon}\right) \psi \eta d x+\int \epsilon R \eta d x \\
\leq & \frac{\|\eta\|^{2}}{16 \epsilon^{2}}+C \epsilon^{4}+C(1+D)\left(\|\psi\|^{2}+\|\eta\|^{2}+\|\nabla \eta\|^{2}\right) .
\end{aligned}
$$

Moreover, noting that

$$
f\left(w_{\epsilon}^{2}\right)-f\left(w^{\epsilon 2}\right)=\left(w_{\epsilon}^{2}-w^{\epsilon 2}\right) \int_{0}^{1} f^{\prime}\left(w_{\epsilon}^{2}+\sigma\left(w_{\epsilon}^{2}-w^{\epsilon 2}\right)\right) d \sigma
$$

one has

$$
\begin{aligned}
\left\|f\left(w_{\epsilon}^{2}\right)-f\left(w^{\epsilon 2}\right)\right\| & \leq C\|\psi\|\left\|\int_{0}^{1} f^{\prime}\left(w_{\epsilon}^{2}+\sigma\left(w_{\epsilon}^{2}-w^{\epsilon 2}\right)\right) d \sigma\right\|_{4} \\
& \leq C\|\psi\| \int_{0}^{1}\left\|f^{\prime}\left(w_{\epsilon}^{2}+\sigma\left(w_{\epsilon}^{2}-w^{\epsilon 2}\right)\right)\right\|_{4} d \sigma \\
& \leq C\|\psi\| \int_{0}^{1}\left(1+\left\|\sigma\left(w_{\epsilon}^{2}-w^{\epsilon 2}\right)\right\|_{4}\right) d \sigma \\
& \leq C\left(1+D^{5}\right)\|\psi\| .
\end{aligned}
$$

So, we can obtain

$$
\frac{1}{\epsilon} \int \nabla\left(f\left(w_{\epsilon}^{2}\right)-f\left(w^{\epsilon 2}\right)\right) \eta d x \leq \frac{\|\operatorname{div} \eta\|^{2}}{16 \epsilon^{2}}+C\left(1+D^{10}\right)\|\psi\|^{2} .
$$

Using integration by parts, we can compute

$$
\begin{aligned}
\frac{\epsilon}{2} \int \nabla\left(\frac{\Delta w_{\epsilon}}{w_{\epsilon}}-\frac{\Delta w^{\epsilon}}{w^{\epsilon}}\right) \eta d x & =-\frac{\epsilon}{2} \int\left(\frac{w^{\epsilon} \Delta \psi-\psi \Delta w^{\epsilon}}{w_{\epsilon} w^{\epsilon}}\right) \operatorname{div} \eta d x \\
& \leq \frac{\|\operatorname{div} \eta\|^{2}}{16 \epsilon^{2}}+C(1+D)\left(\|\psi\|^{2}+\epsilon^{2}\|\Delta \psi\|^{2}\right)
\end{aligned}
$$

Insertion of the above inequalities into (3.7) yields

$$
\begin{aligned}
& \frac{1}{2} \frac{d}{d t} \int \eta^{2} d x+\frac{15}{16 \epsilon^{2}} \int \eta^{2} d x \leq \frac{\|\operatorname{div} \eta\|^{2}}{8 \epsilon^{2}}+C \epsilon^{4} \\
&+\quad C\left(1+D^{10}\right)\left(\|(\eta, \nabla \eta)\|^{2}+\|\psi\|^{2}+\|\nabla \psi\|^{2}+\epsilon^{2}\|\Delta \psi\|^{2}\right) .
\end{aligned}
$$

Similarly, taking $\partial_{x}^{\alpha}(4 \geq \alpha \geq 1)$ and in a completely similar way, we can obtain

$$
\begin{aligned}
& \frac{1}{2} \frac{d}{d t} \int\left(\partial_{x}^{\alpha} \eta\right)^{2} d x+\frac{15}{16 \epsilon^{2}} \int\left(\partial_{x}^{\alpha} \eta\right)^{2} d x \leq \frac{\left\|\partial_{x}^{\alpha} \operatorname{div} \eta\right\|^{2}}{8 \epsilon^{2}}+C \epsilon^{4} \\
+\quad & C\left(1+D^{10}\right)\left(\left\|\partial_{x}^{\alpha} \Delta \psi\right\|^{2}+\|(\psi, \eta)\|_{|\alpha|+1}^{2}\right) .
\end{aligned}
$$

Moreover, from (3.5), we can deduce that

$$
\|\operatorname{div} \eta\|^{2} \leq 4 \epsilon^{2}\left\|\psi_{t}\right\|+C \epsilon^{2}\|\eta\|^{2}+\epsilon^{2}\left(1+D^{2}\right)\|\nabla \psi\|^{2}+C\|\eta\|^{2}
$$

and

$$
\left\|\partial_{x}^{\alpha} \operatorname{div} \eta\right\|^{2} \leq 4 \epsilon^{2}\left\|\partial_{x}^{\alpha} \psi_{t}\right\|+C \epsilon^{2}\|\eta\|_{|\alpha|}^{2}+\epsilon^{2}\left(1+D^{2}\right)\|\nabla \psi\|_{|\alpha|}^{2}+C\|\eta\|_{|\alpha|}^{2}
$$


Therefore, combining (3.12), (3.13) and (3.14), we can establish (3.6). This completes the proofs.

LEmma 3.3. Under the assumptions of Theorem 1.1, we have for $0 \leq \alpha \leq 4$,

$$
\begin{aligned}
& \frac{d}{d t} \int\left(\frac{1}{2 \epsilon^{2}}\left(\partial_{x}^{\alpha} \psi\right)^{2}+\partial_{x}^{\alpha} \psi \partial_{x}^{\alpha} \psi_{t}+\left(\partial_{x}^{\alpha} \psi_{t}\right)^{2}+\frac{1}{4}\left(\partial_{x}^{\alpha} \Delta \psi\right)^{2}\right) d x+\frac{1}{4} \int\left(\partial_{x}^{\alpha} \Delta \psi\right)^{2} d x \\
+ & \frac{d}{d t} \int \frac{p^{\prime}\left(w_{\epsilon}^{2}+\sigma\left(w_{\epsilon}^{2}-w^{\epsilon 2}\right)\right)\left(w_{\epsilon}+w^{\epsilon}\right)}{2 \epsilon^{2} w_{\epsilon}} \partial_{x}^{\alpha}(\nabla \psi)^{2} d x \\
& +\left(\frac{27}{16 \epsilon^{2}}-\frac{17}{16}\right) \int\left(\partial_{x}^{\alpha} \psi_{t}\right)^{2} d x+\frac{1}{2 \epsilon^{2}} \int \frac{p^{\prime}\left(w_{\epsilon}^{2}+\sigma\left(w_{\epsilon}^{2}-w^{\epsilon 2}\right)\right)\left(w_{\epsilon}+w^{\epsilon}\right)}{w_{\epsilon}}\left(\partial_{x}^{\alpha} \nabla \psi\right)^{2} d x \\
\leq & C\left(1+D^{3}\right)\left(\left\|\partial_{x}^{\alpha} \Delta \psi\right\|^{2}+\left\|\partial_{x}^{\alpha} \psi_{t}\right\|^{2}\right)+\frac{C}{\epsilon^{2}}\left(\|\psi\|_{1+|\alpha|}^{2}+\|\eta\|_{1+|\alpha|}^{2}\right)+C \epsilon^{2} .
\end{aligned}
$$

Proof. Multiplying (3.3) by $\psi$ and integrating the resultant equation over $\Omega$, one has

$$
\begin{aligned}
& \frac{d}{d t} \int\left(\psi \psi_{t}+\frac{1}{2 \epsilon^{2}} \psi^{2}\right) d x-\int \psi_{t}^{2} d x+\frac{1}{4} \int(\Delta \psi)^{2} d x+\int\left(\frac{1}{w_{\epsilon}} w_{\epsilon t}^{2}-\frac{1}{w^{\epsilon}} w_{t}^{\epsilon 2}\right. \\
- & \frac{1}{2 \epsilon^{2} w_{\epsilon}} \Delta\left(p\left(w_{\epsilon}^{2}\right)-p\left(w^{\epsilon 2}\right)\right)+\nabla^{2} \cdot\left(w_{\epsilon}^{2} u_{\epsilon} \otimes u_{\epsilon}-w^{\epsilon 2} u^{\epsilon} \otimes u^{\epsilon}\right) \\
+ & \frac{1}{2 \epsilon^{2} w_{\epsilon}} \operatorname{div}\left(w_{\epsilon}^{2} \nabla \Delta^{-1}\left(w_{\epsilon}^{2}-\epsilon b(x)\right)\right)-\frac{1}{2 \epsilon^{2} w^{\epsilon}} \operatorname{div}\left(w^{\epsilon 2} \nabla \Delta^{-1}\left(w^{\epsilon 2}-b(x)\right)\right) \\
- & \left.\frac{1}{4 w_{\epsilon}}\left(\Delta w_{\epsilon}\right)^{2}+\frac{1}{4 w^{\epsilon}}\left(\Delta w^{\epsilon}\right)^{2}\right) \psi d x=-\frac{1}{2} \int \operatorname{div} R \cdot \psi d x .
\end{aligned}
$$

For the integral terms in (3.16), from Cauchy-Schwarz's inequalities, we have

$$
\begin{aligned}
& -\int\left(\frac{1}{w_{\epsilon}} w_{\epsilon t}^{2}-\frac{1}{w^{\epsilon}} w_{t}^{\epsilon 2}\right) \psi d x-\frac{1}{2} \int \operatorname{div} R \cdot \psi d x \\
= & -\int \frac{-\left(w_{\epsilon t}\right)^{2} \psi+w_{\epsilon}\left(w_{\epsilon}+w^{\epsilon}\right)_{t} \psi_{t}}{w_{\epsilon} w^{\epsilon}} \psi d x-\frac{1}{2} \int \operatorname{div} R \cdot \psi d x \\
\leq & \frac{1}{16}\left\|\psi_{t}\right\|^{2}+\frac{C(1+D)}{\epsilon^{2}}\|\psi\|^{2}+C \epsilon^{2}
\end{aligned}
$$

and

$$
\begin{aligned}
& -\frac{1}{4} \int\left(\frac{\left(\Delta w_{\epsilon}\right)^{2}}{w_{\epsilon}}-\frac{\left(\Delta w^{\epsilon}\right)^{2}}{w^{\epsilon}}\right) \psi d x+\frac{1}{2 \epsilon^{2}} \int\left(\frac{\operatorname{div}\left(w_{\epsilon}^{2} \nabla \Delta^{-1}\left(w_{\epsilon}^{2}-b(x)\right)\right)}{w_{\epsilon}}\right. \\
& \left.-\frac{\operatorname{div}\left(w^{\epsilon 2} \nabla \Delta^{-1}\left(w^{\epsilon 2}-b(x)\right)\right)}{w^{\epsilon}}\right) \psi d x \\
= & -\frac{1}{4 \epsilon^{2}} \int \frac{-\left(\Delta w_{\epsilon}\right)^{2} \psi+w_{\epsilon}\left(\Delta w_{\epsilon}+\Delta w^{\epsilon}\right) \Delta \psi}{w_{\epsilon} w^{\epsilon}} \psi d x \\
& +\frac{1}{2 \epsilon^{2}} \int \frac{2 w_{\epsilon} \nabla w_{\epsilon} \nabla \Delta^{-1}\left(w_{\epsilon}^{2}-b(x)\right)+w_{\epsilon}^{2}\left(w_{\epsilon}^{2}-b(x)\right)}{w_{\epsilon}} \\
& -\frac{2 w^{\epsilon} \nabla w^{\epsilon} \nabla \Delta^{-1}\left(w^{\epsilon 2}-b(x)\right)+w^{\epsilon 2}\left(w_{\epsilon}^{2}-b(x)\right)}{w^{\epsilon}} \psi d x \\
\leq & \frac{C\left(1+D^{2}\right)}{\epsilon^{2}}\left(\|\psi\|^{2}+\|\nabla \psi\|^{2}+\epsilon^{2}\|\Delta \psi\|^{2}\right) .
\end{aligned}
$$


Similarly, integration by parts and Young's inequalities lead to

$$
\begin{aligned}
& -\frac{1}{2 \epsilon^{2}} \int\left(\frac{\nabla^{2} \cdot\left(w_{\epsilon}^{2} u_{\epsilon} \otimes u_{\epsilon}\right)}{w_{\epsilon}}-\frac{\nabla^{2} \cdot\left(w^{\epsilon 2} u^{\epsilon} \otimes u^{\epsilon}\right)}{w^{\epsilon}}\right) \psi d x \\
= & -\frac{1}{2 \epsilon^{2}} \int\left(\frac{\nabla^{2}\left(w_{\epsilon}^{2}\right) u_{\epsilon}^{2}+2 \nabla\left(w_{\epsilon}^{2}\right) \nabla \cdot\left(u_{\epsilon}^{2}\right)+w_{\epsilon}^{2} \nabla^{2} \cdot\left(u_{\epsilon}^{2}\right)}{w_{\epsilon}}\right. \\
& \left.-\frac{\nabla^{2}\left(w^{\epsilon 2}\right) u^{\epsilon 2}+2 \nabla\left(w^{\epsilon 2}\right) \nabla \cdot\left(u^{\epsilon 2}\right)+w^{\epsilon 2} \nabla^{2} \cdot\left(u^{\epsilon 2}\right)}{w^{\epsilon}}\right) \psi d x \\
\leq & \frac{C\left(1+D^{3}\right)}{\epsilon^{2}}\left(\|\psi\|^{2}+\|\nabla \psi\|^{2}+\epsilon^{2}\|\Delta \psi\|^{2}+\|\eta\|^{2}+\|\nabla \eta\|^{2}\right) .
\end{aligned}
$$

Similar to (3.9), we have

$$
\begin{aligned}
& -\frac{1}{2 \epsilon^{2}} \int\left(\frac{\Delta p\left(w_{\epsilon}^{2}\right)}{w_{\epsilon}}-\frac{\Delta p\left(w^{\epsilon 2}\right)}{w^{\epsilon}}\right) \psi d x \\
= & -\frac{1}{2 \epsilon^{2}} \int\left(\frac{1}{w^{\epsilon}} \Delta\left(p\left(w_{\epsilon}^{2}\right)-p\left(w^{\epsilon 2}\right)\right)+\Delta p\left(w_{\epsilon}^{2}\right)\left(\frac{1}{w_{\epsilon}}-\frac{1}{w^{\epsilon}}\right)\right) \psi d x \\
= & \frac{1}{2 \epsilon^{2}} \int \nabla\left(\frac{\psi}{w^{\epsilon}}\right) \nabla\left(p\left(w_{\epsilon}^{2}\right)-p\left(w^{\epsilon 2}\right)\right) d x+\frac{1}{2 \epsilon^{2}} \int \frac{\Delta p\left(w_{\epsilon}^{2}\right)}{w_{\epsilon} w^{\epsilon}} \psi^{2} d x \\
\leq & \int \frac{p^{\prime}\left(w_{\epsilon}^{2}+\sigma\left(w_{\epsilon}^{2}-w^{\epsilon 2}\right)\right)\left(w_{\epsilon}+w^{\epsilon}\right)}{2 \epsilon^{2} w_{\epsilon}}(\nabla \psi)^{2} d x+\frac{C\left(1+D^{2}\right)}{\epsilon^{2}}\left(\|\psi\|^{2}+\|\nabla \psi\|^{2}\right) .
\end{aligned}
$$

Therefore, we have

$$
\begin{aligned}
& \frac{d}{d t} \int\left(\psi \psi_{t}+\frac{1}{2 \epsilon^{2}} \psi^{2}\right) d x-\frac{17}{16} \int \psi_{t}^{2} d x+\frac{1}{4} \int(\Delta \psi)^{2} d x \\
& +\frac{1}{2 \epsilon^{2}} \int \frac{p^{\prime}\left(w_{\epsilon}^{2}+\sigma\left(w_{\epsilon}^{2}-w^{\epsilon 2}\right)\right)\left(w_{\epsilon}+w^{\epsilon}\right)}{w_{\epsilon}}(\nabla \psi)^{2} d x \\
\leq & \frac{C\left(1+D^{10}\right)}{\epsilon^{2}}\|(\psi, \eta)\|_{1}^{2}+C\left(1+D^{3}\right)\|\Delta \psi\|^{2}+C \epsilon^{2} .
\end{aligned}
$$

Next, multiplying (3.3) by $2 \psi_{t}$ and integrating the resultant equation over $\Omega$, one has

$$
\begin{aligned}
& \frac{d}{d t} \int\left(\psi_{t}^{2}+\frac{1}{4}(\Delta \psi)^{2}\right) d x+\frac{2}{\epsilon^{2}} \int \psi_{t}^{2} d x+2 \int\left(\frac{1}{w_{\epsilon}} w_{\epsilon t}^{2}-\frac{1}{w^{\epsilon}} w_{t}^{\epsilon 2}-\frac{1}{2 \epsilon^{2} w_{\epsilon}} \Delta p\left(w_{\epsilon}^{2}\right)\right. \\
+ & \frac{1}{2 \epsilon^{2} w^{\epsilon}} \Delta p\left(w^{\epsilon 2}\right)-\frac{1}{2 \epsilon^{2} w_{\epsilon}} \nabla^{2}\left(w_{\epsilon}^{2} u_{\epsilon} \otimes u_{\epsilon}\right)+\frac{1}{2 \epsilon^{2} w^{\epsilon}} \nabla^{2}\left(w^{\epsilon 2} u^{\epsilon} \otimes u^{\epsilon}\right) \\
+ & \frac{1}{2 \epsilon^{2} w_{\epsilon}} \operatorname{div}\left(w_{\epsilon}^{2} \nabla \Delta^{-1}\left(w_{\epsilon}^{2}-b(x)\right)\right)-\frac{1}{2 \epsilon^{2} w^{\epsilon}} \operatorname{div}\left(w^{\epsilon 2} \nabla \Delta^{-1}\left(w^{\epsilon 2}-b(x)\right)\right) \\
- & \left.\frac{1}{4 w_{\epsilon}}\left(\Delta w_{\epsilon}\right)^{2}+\frac{1}{4 w^{\epsilon}}\left(\Delta w^{\epsilon}\right) 2\right) \psi_{t} d x=-\int \operatorname{div} R \psi_{t} d x .
\end{aligned}
$$


In completely the same way, we have

$$
\begin{aligned}
& 2 \int\left(\frac{1}{w_{\epsilon}} w_{\epsilon t}^{2}-\frac{1}{w^{\epsilon}} w_{t}^{\epsilon 2}\right) \psi_{t} d x+\int \operatorname{div} R \psi_{t} d x-\frac{1}{2} \int\left(\frac{\left(\Delta w_{\epsilon}\right)^{2}}{w_{\epsilon}}-\frac{\left(\Delta w^{\epsilon}\right)^{2}}{w^{\epsilon}}\right) \psi_{t} d x \\
& +\frac{1}{\epsilon^{2}} \int\left(\frac{\operatorname{div}\left(w_{\epsilon}^{2} \Delta^{-1}\left(w_{\epsilon}^{2}-b(x)\right)\right)}{w_{\epsilon}}-\frac{\operatorname{div}\left(w^{\epsilon 2} \Delta^{-1}\left(w^{\epsilon 2}-b(x)\right)\right)}{w^{\epsilon}}\right) \psi_{t} d x \\
= & \int \frac{w^{\epsilon}\left(w_{\epsilon}+w^{\epsilon}\right)_{t} \psi_{t}+\left(w_{t}^{\epsilon}\right)^{2} \psi}{w_{\epsilon} w^{\epsilon}} \psi_{t} d x+\int \operatorname{div} R \psi_{t} d x-\frac{1}{2 \epsilon^{2}} \int\left(\frac{\Delta w_{\epsilon}^{2}}{w_{\epsilon}}-\frac{\Delta w^{\epsilon 2}}{w^{\epsilon}}\right) \psi_{t} d x \\
& +\frac{1}{\epsilon^{2}} \int\left(\frac{\operatorname{div}\left(w_{\epsilon}^{2} \Delta^{-1}\left(w_{\epsilon}^{2}-b(x)\right)\right)}{w_{\epsilon}}-\frac{\operatorname{div}\left(w^{\epsilon 2} \Delta^{-1}\left(w^{\epsilon 2}-b(x)\right)\right)}{w^{\epsilon}}\right) \psi_{t} d x \\
\leq & C\left(1+D^{2}\right)\left\|\left(\psi, \psi_{t}\right)\right\|^{2}+\frac{1}{8 \epsilon^{2}}\left\|\psi_{t}\right\|^{2}+\frac{C(1+D)}{\epsilon^{2}}\left(\|\psi\|^{2}+\epsilon^{2}\|\Delta \psi\|^{2}\right)+C \epsilon^{2} .
\end{aligned}
$$

Moreover, with the aid of integration by parts, we have

$$
\begin{aligned}
& -\frac{1}{\epsilon^{2}} \int\left(\frac{\Delta p\left(w_{\epsilon}^{2}\right)}{w_{\epsilon}}-\frac{\Delta p\left(w^{\epsilon 2}\right)}{w^{\epsilon}}\right) \psi_{t} d x \\
= & \frac{1}{\epsilon^{2}} \int\left(\nabla\left(\frac{1}{w_{\epsilon}}\right) \nabla\left(p\left(w_{\epsilon}^{2}\right)-p\left(w^{\epsilon} 2\right)\right)_{x} \psi_{t}+\frac{1}{w_{\epsilon}} p^{\prime}\left(w_{\epsilon}^{2}+\sigma\left(w_{\epsilon}^{2}-w^{\epsilon 2}\right)\right)\left(w_{\epsilon}+w^{\epsilon}\right)\left((\nabla \psi)^{2}\right)_{t}\right) d x \\
\geq & \frac{d}{d t} \int \frac{p^{\prime}\left(w_{\epsilon}^{2}+\sigma\left(w_{\epsilon}^{2}-w^{\epsilon 2}\right)\right)\left(w_{\epsilon}+w^{\epsilon}\right)}{2 \epsilon^{2} w_{\epsilon}}(\nabla \psi)^{2} d x-\frac{C\left(1+D^{10}\right)}{\epsilon^{2}}\|\psi\|_{1}^{2}-\frac{1}{16 \epsilon^{2}}\left\|\psi_{t}\right\|^{2}
\end{aligned}
$$

and

$$
\begin{aligned}
& -\frac{1}{\epsilon^{2}} \int\left(\frac{\nabla^{2}\left(w_{\epsilon}^{2} u_{\epsilon} \otimes u_{\epsilon}\right)}{w_{\epsilon}}-\frac{\nabla^{2}\left(w^{\epsilon 2} u^{\epsilon} \otimes u^{\epsilon}\right)}{w^{\epsilon}}\right) \psi_{t} d x \\
= & -\frac{1}{\epsilon^{2}} \int \frac{\nabla^{2}\left(w_{\epsilon}^{2}\right) u_{\epsilon}^{2}+2 \nabla\left(w_{\epsilon}^{2}\right) \nabla\left(u_{\epsilon}^{2}\right)+w_{\epsilon}^{2} \nabla^{2}\left(u_{\epsilon}^{2}\right)}{w_{\epsilon}} \\
& \left.-\frac{\nabla^{2}\left(w^{\epsilon 2}\right) u^{\epsilon 2}+2 \nabla\left(w^{\epsilon 2}\right) \nabla\left(u^{\epsilon 2}\right)+w^{\epsilon 2} \nabla^{2}\left(u^{\epsilon 2}\right)}{w^{\epsilon}}\right) \psi_{t} d x \\
\leq & -\frac{2}{\epsilon^{2}} \int \frac{w_{\epsilon}^{2} w^{\epsilon} u_{\epsilon} \nabla^{2} u_{\epsilon}-w^{\epsilon 2} w_{\epsilon} u^{\epsilon} \nabla^{2} u^{\epsilon}}{w_{\epsilon} w^{\epsilon}} \psi_{t} d x+\frac{1}{16 \epsilon^{2}}\left\|\psi_{t}\right\|^{2} \\
& +\frac{C\left(1+D^{3}\right)}{\epsilon^{2}}\left(\|\psi\|^{2}+\|\nabla \psi\|^{2}+\epsilon^{2}\left\|\nabla^{2} \psi\right\|^{2}+\|\eta\|^{2}+\|\nabla \eta\|^{2}\right) \\
\leq & -\frac{2}{\epsilon^{2}} \int \frac{-u_{\epsilon} \nabla^{2} u_{\epsilon} w_{\epsilon}^{2} \psi+w_{\epsilon}^{2} w^{\epsilon} \nabla^{2} u_{\epsilon} \eta+w_{\epsilon} \nabla^{2} u_{\epsilon} u^{\epsilon}\left(w_{\epsilon}^{2}-w^{\epsilon 2}\right)+u^{\epsilon} w_{\epsilon} w^{\epsilon 2} \nabla^{2} \eta}{w_{\epsilon} w^{\epsilon}} \psi_{t} d x \\
& +\frac{1}{16 \epsilon^{2}}\left\|\psi_{t}\right\|^{2}+\frac{C\left(1+D^{3}\right)}{\epsilon^{2}}\left(\|\psi\|^{2}+\|\nabla \psi\|^{2}+\epsilon^{2}\left\|\nabla^{2} \psi\right\|^{2}+\|\eta\|^{2}+\|\nabla \eta\|^{2}\right) \\
\leq & \frac{4}{\epsilon} \int u^{\epsilon} w^{\epsilon} \operatorname{div}\left(\frac{1}{w^{\epsilon}}\left(\psi_{t}+\frac{1}{2 \epsilon} \eta u_{\epsilon x}+\frac{1}{\epsilon} u^{\epsilon} \nabla \psi+\frac{1}{\epsilon} \eta \nabla w_{\epsilon}\right)\right) \psi_{t} d x \\
& +\frac{1}{16 \epsilon^{2}}\left\|\psi_{t}\right\|^{2}+\frac{C\left(1+D^{3}\right)}{\epsilon^{2}}\left(\|\psi\|^{2}+\|\nabla \psi\|^{2}+\epsilon^{2}\left\|\nabla^{2} \psi\right\|^{2}+\|\eta\|^{2}+\|\nabla \eta\|^{2}\right) \\
\leq \quad & C\left(1+D^{3}\right)\left\|\psi_{t}\right\|^{2}+\frac{1}{8 \epsilon^{2}}\left\|\psi_{t}\right\|^{2} \\
& +\frac{C\left(1+D^{3}\right)}{\epsilon^{2}}\left(\|\psi\|^{2}+\|\nabla \psi\|^{2}+\epsilon^{2}\left\|\nabla^{2} \psi\right\|^{2}+\|\eta\|^{2}+\|\nabla \eta\|^{2}\right) .
\end{aligned}
$$


Further, one has

$$
\begin{aligned}
& \frac{d}{d t} \int\left(\psi_{t}^{2}+\frac{p^{\prime}\left(w_{\epsilon}^{2}+\sigma\left(w_{\epsilon}^{2}-w^{\epsilon 2}\right)\right)\left(w_{\epsilon}+w^{\epsilon}\right)}{2 \epsilon^{2} w_{\epsilon}}(\nabla \psi)^{2}+\frac{1}{4}(\Delta \psi)^{2}\right) d x+\frac{27}{16 \epsilon^{2}} \int \psi_{t}^{2} d x \\
\leq & C\left(1+D^{3}\right)\left\|\left(\psi, \psi_{t}\right)\right\|^{2}+\frac{C\left(1+D^{10}\right)}{\epsilon^{2}}\left(\epsilon^{2}\|\Delta \psi\|^{2}+\|(\psi, \eta)\|_{1}^{2}\right)+C \epsilon^{2} .
\end{aligned}
$$

Combining (3.17) and (3.18) leads to

$$
\begin{aligned}
& \frac{d}{d t} \int\left(\frac{1}{2 \epsilon^{2}} \psi^{2}+\psi \psi_{t}+\psi_{t}^{2}+\frac{p^{\prime}\left(w_{\epsilon}^{2}+\sigma\left(w_{\epsilon}^{2}-w^{\epsilon 2}\right)\right)\left(w_{\epsilon}+w^{\epsilon}\right)}{2 \epsilon^{2} w_{\epsilon}}(\nabla \psi)^{2}+\frac{1}{4}(\Delta \psi)^{2}\right) d x \\
& +\left(\frac{27}{16 \epsilon^{2}}-\frac{17}{16}\right) \int \psi_{t}^{2} d x+\int\left(\frac{1}{4}(\Delta \psi)^{2}+\frac{p^{\prime}\left(w_{\epsilon}^{2}+\sigma\left(w_{\epsilon}^{2}-w^{\epsilon 2}\right)\right)\left(w_{\epsilon}+w^{\epsilon}\right)}{2 \epsilon^{2} w_{\epsilon}}(\nabla \psi)^{2}\right) d x \\
\leq & C\left(1+D^{3}\right)\left\|\left(\psi, \psi_{t}\right)\right\|^{2}+\frac{C\left(1+D^{10}\right)}{\epsilon^{2}}\left(\epsilon^{2}\|\Delta \psi\|^{2}+\|(\psi, \eta)\|_{1}^{2}\right)+C \epsilon^{2} .
\end{aligned}
$$

In order to obtain a higher-order estimate, we differentiate with respect to $x$; therefore by repeating the previous steps, we have

$$
\begin{aligned}
& \frac{d}{d t} \int\left(\frac{1}{2 \epsilon^{2}}\left(\partial_{x}^{\alpha} \psi\right)^{2}+\partial_{x}^{\alpha} \psi \partial_{x}^{\alpha} \psi_{t}+\left(\partial_{x}^{\alpha} \psi_{t}\right)^{2}+\frac{1}{4 \epsilon^{2}}\left(\partial_{x}^{\alpha} \Delta \psi\right)^{2}\right) d x \\
+ & \frac{d}{d t} \int \frac{p^{\prime}\left(w_{\epsilon}^{2}+\sigma\left(w_{\epsilon}^{2}-w^{\epsilon 2}\right)\right)\left(w_{\epsilon}+w^{\epsilon}\right)}{2 \epsilon^{2} w_{\epsilon}}\left(\partial_{x}^{\alpha} \nabla \psi\right)^{2} d x \\
& +\left(\frac{27}{16 \epsilon^{2}}-\frac{17}{16}\right) \int\left(\partial_{x}^{\alpha} \psi_{t}\right)^{2} d x+\int \frac{p^{\prime}\left(w_{\epsilon}^{2}+\sigma\left(w_{\epsilon}^{2}-w^{\epsilon 2}\right)\right)\left(w_{\epsilon}+w^{\epsilon}\right)}{2 \epsilon^{2} w_{\epsilon}}\left(\partial_{x}^{\alpha} \nabla \psi\right)^{2} d x \\
\leq & C\left(1+D^{3}\right)\left\|\left(\partial_{x}^{\alpha} \psi, \partial_{x}^{\alpha} \psi_{t}\right)\right\|^{2}+\frac{C\left(1+D^{10}\right)}{\epsilon^{2}}\left(\epsilon^{2}\left\|\partial_{x}^{\alpha} \Delta \psi\right\|^{2}+\|(\psi, \eta)\|_{|\alpha|+1}^{2}\right)+C \epsilon^{2} .
\end{aligned}
$$

This completes the proofs of Lemma 3.3 .

Combining (3.6) and (3.20) yields

$$
\begin{aligned}
& \frac{d}{d t}\left(\left\|\left(\partial_{x}^{\alpha} \psi, \partial_{x}^{\alpha} \eta, \epsilon \partial_{x}^{\alpha} \Delta \psi\right)\right\|^{2}+\epsilon^{2}\left\|\partial_{x}^{\alpha} \psi_{t}\right\|^{2}\right)+\frac{1}{\epsilon^{2}}\left\|\left(\epsilon \partial_{x}^{\alpha} \Delta \psi, \epsilon \partial_{x}^{\alpha} \psi_{t}, \partial_{x}^{\alpha} \eta\right)\right\|^{2} \\
\leq & C \epsilon^{4}+C\left(1+D^{10}\right)\left(\|(\psi, \eta)\|_{|\alpha|+1}^{2}+\epsilon^{2}\left\|\partial_{x}^{\alpha} \psi_{t}\right\|^{2}+\epsilon\left\|\partial_{x}^{\alpha} \Delta \psi\right\|^{2}\right) .
\end{aligned}
$$

Then we integrate (3.21) from 0 to $T$ with $[0, T] \subset\left[0, \min \left\{T_{\epsilon}, T_{*}\right\}\right)$ to obtain

$$
\begin{aligned}
& \left(\left\|\left(\partial_{x}^{\alpha} \psi, \partial_{x}^{\alpha} \eta, \epsilon \partial_{x}^{\alpha} \Delta \psi\right)\right\|^{2}+\epsilon^{2}\left\|\partial_{x}^{\alpha} \psi_{t}\right\|^{2}\right)+\int_{0}^{T} \frac{1}{\epsilon^{2}}\left\|\left(\epsilon \partial_{x}^{\alpha} \Delta \psi, \epsilon \partial_{x}^{\alpha} \psi_{t}, \partial_{x}^{\alpha} \eta\right)\right\|^{2} d t \\
\leq & C T \epsilon^{4}+C \int_{0}^{T}\left(1+D^{10}\right)\left(\|(\psi, \eta)\|_{\alpha+1}^{2}+\epsilon^{2}\left\|\partial_{x}^{\alpha} \psi_{t}\right\|^{2}+\epsilon^{2}\left\|\partial_{x}^{\alpha} \Delta \psi\right\|^{2}\right) d t .
\end{aligned}
$$

Here we have used the fact that the initial data are in equilibrium. Summing up the last inequality over all $\alpha$ satisfying $|\alpha| \leq 2$, and noting that

$$
\left\|\partial_{x}^{5} \psi\right\| \leq C\left(\left\|\partial_{x}^{4} \psi\right\|+\left\|\partial_{x}^{6} \psi\right\|\right),
$$


and using (3.14), we get

$$
\begin{aligned}
& \|\psi\|_{4}^{2}+\epsilon^{2}\left\|\partial_{x}^{5} \psi\right\|_{1}^{2}+\epsilon^{2}\left\|\psi_{t}\right\|_{4}^{2}+\|\eta\|_{5}^{2} \\
& +\frac{1}{\epsilon^{2}} \int_{0}^{T}\left(\|\psi\|_{4}^{2}+\epsilon^{2}\left\|\partial_{x}^{5} \psi\right\|_{1}^{2}+\epsilon^{2}\left\|\psi_{t}\right\|_{4}^{2}+\|\eta\|_{5}^{2}\right) d t \\
\leq & C T_{*} \epsilon^{4}+C \int_{0}^{T}\left(1+D^{10}\right)\left(\|\psi\|_{4}^{2}+\epsilon^{2}\left\|\partial_{x}^{5} \psi\right\|_{1}^{2}+\epsilon^{2}\left\|\psi_{t}\right\|_{4}^{2}+\|\eta\|_{5}^{2}\right) d t .
\end{aligned}
$$

We apply Gronwall's lemma to (3.22) to get

$$
\|\psi\|_{4}^{2}+\epsilon^{2}\left\|\partial_{x}^{5} \psi\right\|_{1}^{2}+\|\eta\|_{5}^{2}+\epsilon^{2}\left\|\psi_{t}\right\|_{4}^{2} \leq C T_{*} \epsilon^{4} \exp \left[C \int_{0}^{T}\left(1+D^{10}\right) d t\right] .
$$

From the definition of $D(t)$ and (3.23), it follows that

$$
D(T)^{2} \leq C T_{*} \epsilon^{2} \exp \left[C \int_{0}^{T}\left(1+D^{10}\right) d t\right] \equiv \Phi(T),
$$

which implies

$$
\Phi^{\prime}(t)=C\left(1+D^{10}\right) \Phi(t) \leq C \Phi(t)+C \Phi^{6}(t) .
$$

Applying the nonlinear Gronwall-type inequality in 25] to (3.25) yields

$$
\Phi(t) \leq e^{C T_{*}}
$$

for $t \in\left[0, \min \left\{T_{*}, T_{\epsilon}\right\}\right)$ if we choose $\epsilon$ so small that

$$
\Phi(0)=C T_{*} \epsilon^{2} \leq e^{-C T_{*}} .
$$

Further, it follows from (3.24) and (3.26) that there exists a constant $c$, independent of $\epsilon$, such that

$$
D(T) \leq c
$$

for $T \in\left[0, \min \left\{T_{*}, T_{\epsilon}\right\}\right)$. Finally, the theorem is concluded from the definition of $D(t)$, (3.23) and (3.27). This completes the proof of Theorem 1.1.

Acknowledgements. The authors are grateful to the referees for their helpful comments and suggestions on the manuscript. The work is supported in part from the National Science Foundation of China (Grant No. 10701057), the Science Foundation of Shanghai Education Committee (Grant No. 08YZ72), the Shanghai Normal University (Grant No. SK200804), and Leading Academic Discipline Project of Shanghai Normal University (Grant No. DZL707).

\section{REFERENCES}

[1] G. Ali and A. Jüngel, Global smooth solutions to the multi-dimensional hydrodynamic model for two-carrier plasma, J. Differential Equations, 190(2003), 663-685. MR1970046 (2004b:76165)

[2] P. Antonelli and P. Marcati, On the finite energy weak solutions to a system in Quantum Fluid Dynamics, to appear in Comm. Math. Phys., 2008.

[3] F. Brezzi, I. Gasser, P. Markowich and C. Schmeiser, Thermal equilibrium states of the quantum hydrodynamic model for semiconductors in one dimension, Appl. Math. Lett., 8(1995), 47-52. MR 1355150

[4] N. Ben Abdallah and A. Unterreiter, On the stationary quantum drift-diffusion model, Z. Angew. Math. Phys., 49(1998), 251-275. MR.1629183(99j:35178) 
[5] P. Degond and C. Ringhofer, Quantum moment hydrodynamics and the entropy principle, J. Stat. Phys., 112(2003), 587-628. MR.1997263 (2004m:82084)

[6] C. Gardner, The quantum hydrodynamic model for semiconductors devices, SIAM J. Appl. Math., 54(1994), 409-427. MR.1265234 (95a:82120)

[7] I. Gasser, P. A. Markowich, and C. Ringhofer, Closure conditions for classical and quantum moment hierarchies in the small-temperature limit, Transport Theory Stat. Phys., 25(1996), 409-423. MR:1407543 (98c:82059)

[8] I. Gasser and P. A. Markowich, Quantum hydrodynamics, Wigner transforms and the classical limit, Asymptotic Anal., 14(1997), 97-116. MR.1451208 (99i:81043)

[9] I. Gamba and A. Jüngel, Positive solutions to singular second and third order differential equations for quantum fluids, Arch. Rat. Mech. Anal., 156(2001), 183-203. MR.1816474(2002a:82112)

[10] I. Gasser and R. Natalini, The energy transport and the drift diffusion equations as relaxation limits of the hydrodynamic model for semiconductors, Quart. Appl. Math., 57(1999), 269-282. MR1686190 (2000b:82044)

[11] F. Huang, H. -L. Li and A. Matsumura, Existence and stability of steady-state of one-dimensional quantum hydrodynamic system for semiconductors, J. Differential Equations, 225(2006), 1-25. MR:2228690 (2007f:35228)

[12] A. Jüngel, H. -L. Li and A. Matsumura, The relaxation-time limit in the quantum hydrodynamic equations for semiconductors, J. Differential Equations, 225(2006), 440-464. MR2225796 (2007b:82095)

[13] A. Jüngel, Quasi-hydrodynamic semiconductor equations, Progress in Nonlinear Differential Equations, Birkhäuser, 2001. MR1818867(2002h:82083)

[14] A. Jüngel and H. L. Li, Quantum Euler-Poisson system: Existence of stationary states, Arch. Math. (Brno), 40(2004), 435-456. MR2129964 (2005k:35394)

[15] A. Jüngel and H. L. Li, Quantum Euler-Poisson system: Global existence and exponential decay, Quart. Appl. Math., 62(2004), 569-600. MR2086047 (2005g:82078)

[16] T. Luo, R. Natalini and Z. Xin, Large time behavior of the solutions to a hydrodynamic model for semiconductors, SIAM J. Appl. Math., 59-3(1998), 810-830. MR1661255 (2000b:35259)

[17] C. Lattanzio and P. Marcati, The relaxation to the drift-diffusion system for the 3-D isentropic EulerPoisson model for semiconductors, Discrete Contin. Dynam. System, 5(1999), 449-455. MR1665756 (99m:35251)

[18] C. Lattanzio, On the 3-D bipolar isentropic Euler-Poisson model for semiconductors and the driftdiffusion limit, Math. Models Methods Appl. Sci., 10(2000), 351-360. MR1753116 (2001c:82083)

[19] H.-L. Li and P. Marcati, Existence and asymptotic behaviour of multi-dimensional quantum hydrodynamic model for semiconductors, Comm. Math. Phys., 245(2004), 215-247. MR2039696 (2004k:82113)

[20] H.-L. Li and C.-K. Lin, Semiclassical limit and well-posedness of nonlinear Schrödinger-Poisson, Electronic J. Differential Equations, 2003(2003), 1-17.

[21] Y.-P. Li, Diffusion relaxation limit of a nonisentropic hydrodynamic model for semiconductors, Math. Methods Appl. Sci., 30(2007), 2247-2261. MR2363951

[22] P. Marcati and R. Natalini, Weak solutions to a hydrodynamic model for semiconductors and relaxation to the drift-diffusion equation, Arch. Rational Mech. Anal., 129(1995), 129-145. MR1328473 (96b:65088)

[23] P. A. Markowich, C. A. Ringhofev and C. Schmeiser, Semiconductor equations, Springer-Verlag, Wien, New York, 1990. MR1063852 (91j:78011)

[24] A. Unterreiter, The thermal equilibrium solution of a generic bipolar quantum hydrodynamic model, Comm. Math. Phys., 188(1997), 69-88. MR1471332(99c:58050)

[25] W.-A. Yong, Basic aspects of hyperbolic relaxation systems, in Advances in the theory of shock waves, H. Freistuhler and A. Szepessy, eds., Progr. Nonlinear Differential Equations Appl. 47, Birkhäuser Boston, Boston, 2001, 259-305. MR1842777 (2002g:35136)

[26] W.-A. Yong, Relaxation limit of multi-dimensional isentropic hydrodynamical models for semiconductors, SIAM J. Appl. Math., 64(2004), 1737-1748. MR2084208(2006b:35207)

[27] B. Zhang and W. Jerome, On a steady-state quantum hydrodynamic model for semiconductors, Nonlinear Anal., TMA, 26(1996), 845-856. MR1362757 (96i:35105)

[28] C. Zhu and H. Hattori, Stability of steady state solutions for an isentropic hydrodynamic model of semiconductors of two species, J. Differential Equations, 166(2000), 1-32. MR1779253(2001i:82072) 